\title{
Comparison of diagnostic values of ultrasound micro-flow imaging and contrast-enhanced ultrasound for neovascularization in carotid plaques
}

\author{
HONGXUE ZHANG $^{1 *}$, JIANWEN DU ${ }^{1 *}$, HONG WANG $^{1}$, HAILI WANG $^{1}$, \\ JIANHUI JIANG ${ }^{1}$, JINGJIE ZHAO ${ }^{2}$ and HUAN LU ${ }^{1}$ \\ Departments of ${ }^{1}$ UItrasonic Diagnosis and ${ }^{2}$ Pathology, The Second Clinic, \\ Institute of the Chengde Medical College, Chengde, Hebei 067000, P.R. China \\ Received February 16, 2017; Accepted May 17, 2017
}

DOI: $10.3892 /$ etm.2017.4525

\begin{abstract}
The aim of the present study was to compare the diagnostic values of ultrasound micro-flow imaging (SMI) and contrast-enhanced ultrasound (CEUS) for neovascularization in carotid plaques, and to investigate their capacities for predicting the risks of cerebral stroke. A total of 39 patients (64 carotid plaques) with severe carotid artery stenosis undergoing carotid endarterectomy were selected between February 2015 and February 2016, and SMI and CEUS were used to detect neovascularization in plaques. According to the CEUS dynamic graph of plaques, the enhanced intensity visual scales and contrast parameters were obtained. Carotid atherosclerotic plaques were divided into 4 groups. The differences in the enhanced intensity visual scales, contrast parameters, and gray-scale median (GSM) values among the 4 groups were analyzed. Carotid plaque tissue samples from patients were stained for CD34, and the consistency of the methods for the diagnosis of neovascularization in plaques was analyzed. The differences in GSM values, enhanced intensities, and enhanced densities among the 4 groups of plaques were statistically significant $\left(\mathrm{F}=29.365, \chi^{2}=29.025, \chi^{2}=30.871\right.$, $\mathrm{P}<0.001$ ); the differences in enhanced intensities of carotid atherosclerotic plaques with different echo types were statistically significant $\left(\chi^{2}=17.951, \mathrm{P}<0.001\right)$. The enhanced intensity of plaques was negatively correlated with the GSM value $(\mathrm{r}=-0.376, \mathrm{P}<0.01)$, and the enhanced density of plaques was negatively correlated with the GSM value $(\mathrm{r}=-0.252, \mathrm{P}<0.01)$.
\end{abstract}

Correspondence to: Dr Hong Wang, Department of UItrasonic Diagnosis, The Second Clinic, Institute of the Chengde Medical College, North 11 West Street, Chengde, Hebei 067000, P.R. China E-mail: zhx46604@163.com

*Contributed equally

Key words: carotid atherosclerotic plaque, neovascularization, contrast-enhanced ultrasound, ultrasound micro flow imaging, carotid endarterectomy
SMI and CEUS grading had good consistency $(\kappa=0.860>0)$, there were statistically significant differences in new vessel densities with different SMI gradings $(\mathrm{P}<0.001)$, and the clinical symptoms and severity were positively correlated with SMI grading ( $r s=0.592>0$ ). In conclusion, SMI and CEUS have good consistency for evaluating neovascularization in carotid plaques, and have good clinical value for evaluating neovascularization in carotid plaques.

\section{Introduction}

Cerebral stroke is associated with the highest rate of disability in the world, seriously threatening human health (1). Increasing evidence has shown that the incidence rate of ischemic stroke secondary to decreased distal blood flow induced by carotid artery stenosis is not high (2). Similarly, previous findings have shown that the composition of carotid atherosclerosis plaques is closely related to the occurrence of cerebrovascular diseases. Therefore, its pathogenic mechanism is thromboembolism caused by the rupture of vulnerable plaques, rather than vascular lumen stenosis $(1,2)$. The detection and evaluation of morphological changes in plaques may be more clinically valuable than the evaluation of the degree of lumen stenosis. Previous findings showed that vulnerable plaques have larger amounts of eccentric lipids, thinner fibrous caps, substantial inflammatory cell infiltration, intra-plaque hemorrhage, neovascularization, and superficial ulcers (3-5).

Neovascularization in plaques is an independent predictive factor of the rupture of vulnerable plaques, and is currently considered the most potent independent predictive factor of plaque rupture. Previous evidence demonstrated that the development of atherosclerotic plaques may be related to neovascularization (6). In 2005, Virmani et al found that neovascularization in plaques can promote the development of atherosclerotic lesions, and even induce intra-plaque hemorrhage, plaque rupture, and complications, which are important factors related to plaque instability (7). In addition, it was found that the amount of neovascularization in plaques is closely related to clinical manifestations. A higher density of neovascularization is associated with an increased risk of typical clinical manifestations (2-4). Therefore, the detection of 
neovascularization is important for predicting plaque stability, assessing the risk of stroke, and for guiding clinical treatment.

Based on the above observations, new techniques for assessing neovascularization in plaques are necessary. Ultrasound micro-flow imaging (SMI) is a new blood flow imaging technique with high sensitivity, high resolution, and low artifact $(8,9)$. The technique can effectively separate blood flow signals and overlapping tissue motion artifacts by the adaptive calculation method, and accurately detect low-speed blood flow signals (10). However, to the best of our knowlegde, there are no reports on SMI-mediated detection of neovascularization in plaques. Therefore, the aim of the present study was to investigate whether SMI and contrast-enhanced ultrasound (CEUS) are consistent for the detection of neovascularization in plaques that were verified by histology. We further investigated whether clinical symptoms and severities were associated with differences in SMI grading, to provide a basis for early clinical diagnosis and treatment, and reduce the risk of cerebral stroke caused by plaque rupture.

\section{Patients and methods}

Patients. A total of 39 patients (64 carotid atherosclerotic plaques) who underwent cervical vascular examination between February 2015 and February 2016 in the Affiliated Hospital of Chengde Medical College were selected. There were 31 males and 8 females, with an average age of $66.8 \pm 7.4$ years. Informed consent was obtained from all the enrolled patients. There were 11 patients with single carotid atherosclerotic plaques, and 28 patients with multiple carotid atherosclerotic plaques. Inclusion criteria were: patients with carotid atherosclerotic plaques in the common carotid artery and internal carotid artery identified by ultrasound examination (plaque thickness $\geq 2.0 \mathrm{~mm}$, length $\geq 10 \mathrm{~mm}$ ). Exclusion criteria were: patients who were unconscious and could not cooperate in the examination; patients with cardiac insufficiency and severe coronary atherosclerotic cardiopathy, arrhythmia, or allergic constitution; patients with carotid plaques associated with strong echoes with sound shadows, and patients with contraindications for SonoVue (Bracco spA, Milan, Italy).

Instruments and reagents. Aplio 500 ultrasonic diagnostic apparatus (Toshiba, Tokyo, Japan) (11-L4 probe; frequency, 4-11 MHz), and contrast agent, SonoVue were used in the present study.

Ultrasonic examination. Routine ultrasound examination was performed by two experienced physicians in the ultrasound department. Patients were placed in a horizontal position with their head towards the opposite side of examination, with the neck exposed. The carotid artery was scanned by the probe from top to bottom in transverse and longitudinal directions. The largest thickness of plaques was measured in the longitudinal section, and severe carotid stenosis (70-99\%) was determined according to the diagnostic criteria of the maximum systolic velocity (PSV) $>230 \mathrm{~mm} / \mathrm{sec}$, maximum diastolic velocity $(\mathrm{EDV})>100 \mathrm{~mm} / \mathrm{sec}$, PSV in the stenosed segment, distal maximum systolic velocity $>4.0$, and low-speed low-beat change in the distal stenosis spectrum. The probe was kept still, and the SMI mode was turned on to analyze neovascularization in plaques and store images. Examination of neovascularization was conducted again using CEUS, and the mechanical index was reduced to $0.12-0.20$ to avoid the rupture of micro-bubbles at the early stage of angiography. Contrast agent $(5 \mathrm{ml})$ was dissolved in $5 \mathrm{ml}$ of $0.9 \%$ normal saline, followed by intravenous injection of $2.4 \mathrm{ml}$ of contrast agent into the elbow vein. The dose was adjusted according to the specific conditions of the patient (not exceeding $4.8 \mathrm{ml}$ ). The images were stored after continuous observation for $5 \mathrm{~min}$, and $5 \mathrm{ml}$ of $0.9 \%$ normal saline was injected following the contrast agent.

CEUS. Before examination, the patients were asked to minimize body movement and swallowing, and avoid excessive breathing movement. The operator placed the probe on the surface of the body, and chose a clear region of the plaque. The ultrasound contrast mode and double-width real-time display were turned on, simultaneously showing the two-dimensional gray-scale interface and ultrasound contrast interface. The assistant was asked to inject $2.4 \mathrm{ml}$ of contrast agent into the superficial vein in the elbow via bolus injection, immediately followed by $5 \mathrm{ml}$ of $0.9 \%$ normal saline solution. At the same time, the built-in timer and image acquisition were started. During the examination, the probe was maintained to clearly display the sections of interest. After continuous observation for $5 \mathrm{~min}$, and automatic sequence acquisition, the dynamic images were stored in the hard disk. In patients with $\geq 2$ plaques, the interval between examinations was at least $\geq 15 \mathrm{~min}$. If a patient received $\geq 2$ examinations, the built-in timer was restarted after entering the dynamic acquisition interface. After examination, the images were stored in raw data format, followed by in-machine and off-machine quantitative analysis. Intensity-over-time curve (ITC) Qanalysis quantitative software (Nanjing Jiancheng Biotech Co., Nanjing, China) was used for the in-machine analysis: after entering the ITC Qanalysis interface, the continuous dynamic images were opened, and the region of interest (ROI) was drawn manually according to the size and shape of different plaques. Another ROI was drawn in the carotid artery lumen in the proximal part of the plaque as the control, and the ROI was manually adjusted frame-by-frame in the in-machine analysis. The software could automatically generate the ITC in the plaque and arterial lumen.

Carotid plaque grouping. Under conventional two-dimensional gray-scale ultrasound, carotid plaques were divided according to the plaque classification method proposed by Gray-Weale et al (1) combined with the echo of the sternocleidomastoid into homogeneous hypoechoic plaques (Type I): hypoecho in the plaque but with a fibrous cap that could reflect the echo; heterogeneous hypoechoic plaques (Type II): hypoechoic area prevailed in the plaque, and the hyperechoic area was $<25 \%$ of the total plaque area; heterogeneous hyperechoic plaques (Type III): hyperechoic area prevailed in the plaque, and the hypoechoic area was $<25 \%$ of the total plaque area; homogeneous hyperechoic plaques (Type IV): homogeneous hyperechoic plaques; unclassified plaques (Type V): calcified plaques formed with a rear acoustic shadow. Type $\mathrm{V}$ affected the results of analysis because of the rear acoustic 
shadow. Therefore, it was not included in the scope of analysis. Definitions: hypoecho (echo slightly higher than the blood), isoecho (similar to the sternocleidomastoid echo), and hyperecho (higher than the sternocleidomastoid echo).

Gray-scale median (GSM) analysis. Three sections in conventional two-dimensional ultrasound in the longitudinal and transverse directions clearly showed the stored images after plaque echo, and the images were exported using a mobile hard disk. Power Showcase software (Autodesk, Inc., San Rafael, CA, USA) was used to transform the raw data format into JPG format. Adobe Photoshop Elements 11 (Adobe Systems Incorporated, San Jose, CA, USA) was used for image editing to normalize the image processing. The gray-scale range was from 0 (black) to 255 (white), and 2 reference points were selected, blood (0) and adventitia (255). The image was imported to the software, the whole contours of each plaque in the 4 kinds of echoes were obtained using the 'Automatic Selection Tool', and the gray-scale pixel histograms of plaques were obtained. The GSM values of plaques were recorded, and the automatic selection tool was used to draw the local area of the sternocleidomastoid in the same image to obtain the grayscale pixel histogram of the sternocleidomastoid, and the GSM values were recorded. The GSMs of plaques and the sternocleidomastoid in conventional two-dimensional grayscale ultrasound longitudinal and transverse sections were obtained, and the means were compared. Among all plaques, mean GSM values lower than those of the sternocleidomastoid were classified as hypoechoic plaques, while mean GSM values higher than those of the sternocleidomastoid were classified as hyperechoic plaques, and the echo type of the plaque was determined by naked eye examination.

Visual score of CEUS. According to the scoring criterion of Shah et al (6), the degree of development was divided into $0-3$ points: 0 points, no enhancement in plaques; 1 point, point-like enhancement in plaques; 2 points, point-like and 1-2 short line-like enhancements; 3 points, line-like enhancement in plaques throughout the plaque, or with blood flow sign. Double-blind scoring was performed by two physicians; when there was disagreement, the scoring results of the more experienced one prevailed.

Examination method of SMI. The SMI mode was turned on to detect neovascularization in plaques. The sites of neovascularization were divided according to the plaque site into the proximal part, distal part, top part, and basilar part. Neovascularization was the short-line or strip-like hyper echo, although multi-section conventional ultrasound images were required to exclude the calcified echo in plaques. Grading criteria of neovascularization in plaques detected by SMI (5): Grade 0, no blood flow signal in plaques; Grade I, visible blood flow signal in the shoulder or basilar part of plaques; Grade II, visible blood flow signals in the shoulder and basilar part of plaques.

Observational indexes of SMI. The results of the diagnostic methods were recorded, and neovascularization in carotid plaques was divided according to the grading criteria of
Nakamura et al (11): Grade 0, no obvious micro-bubbles in plaques; Grade I, micro-bubbles were limited to one side of the shoulder or outer membrane of plaques; Grade II, visible diffuse micro-bubbles in plaques.

Diagnostic criteria of SMI. According to the unified test standard of the North American Symptomatic Carotid Endarterectomy Trial approved by the ultrasound conference of the Radiological Society of North America (1), the degree of carotid artery stenosis was evaluated. Carotid diameter stenosis was divided into 4 grades: i) mild stenosis, stenosis rate $<50 \%$; ii) moderate stenosis with a stenosis rate of 50-69\%; iii) severe stenosis with a stenosis rate of 70-99\%; and iv) total occlusion with a stenosis rate of $100 \%$.

Statistical analysis. SPSS 17.0 statistical software (IBM Corp., Armonk, NY, USA) was used. The $\kappa$ test was used to test the consistency of grading results of SMI and CEUS. $\kappa>0$ indicated the significance, and larger $\kappa$ value indicated better consistency. One-way ANOVA was used to investigate the differences of neovascularization density in plaques with different SMI grading, demonstrated by CD34 immunohistochemical staining. $\mathrm{P}<0.05$ was considered statistically significant. Spearman's rank correlation test was used to investigate the correlation between SMI and clinical symptoms and severity. The correlation coefficient $r>0$ indicated a positive correlation, $r<0$ indicated a negative correlation, and $r=0$ indicated no correlation.

\section{Results}

Ultrasonic examination results. A total of 39 patients underwent carotid endarterectomy, and 39 carotid plaques were removed. $\kappa$ test result of the consistency of SMI and CEUS: $\kappa=0.860>0$ (Fig. 1; Table I).

Immunohistochemical examination results. A total of 39 patients underwent carotid endarterectomy, and 39 carotid plaques were removed. One-way ANOVA was performed for neovascularization density of immunohistochemical staining in different SMI gradings $(\mathrm{P}<0.001)$. There was no significant difference between Grade 0 and Grade I SMI (P0-I=0.836), while there were significant differences between the other 2 groups (P0-II=0.001, $\mathrm{PI}-\mathrm{II}=0.041)$ (Table II).

Dynamic imaging performances and visual scores of the 4 groups of plaques. A total of 64 carotid plaques from 39 patients were divided into 4 groups according to different echo types, and into 4 grades according to CEUS visual score: $0,1,2$, and 3 points; with $5,23,23$, and 13 , respectively, in each grade (Table III). The consistency rate of the objects was $91 \%(\kappa=0.910, \mathrm{P}<0.001)$. There were statistically significant differences in the enhancement grading and intensity of CEUS visual score among the carotid atherosclerotic plaques with different echo types $\left(\chi^{2}=17.951, \mathrm{P}<0.001\right)$. Lower echo of carotid atherosclerotic plaques was associated with more obvious enhancement of CEUS visual score (Table III). The enhancement scores of the homogeneous hypoechoic plaque group and heterogeneous hypoechoic plaque group were 2 and 3 points, accounting for approximately $66.7 \%(14 / 21)$ 


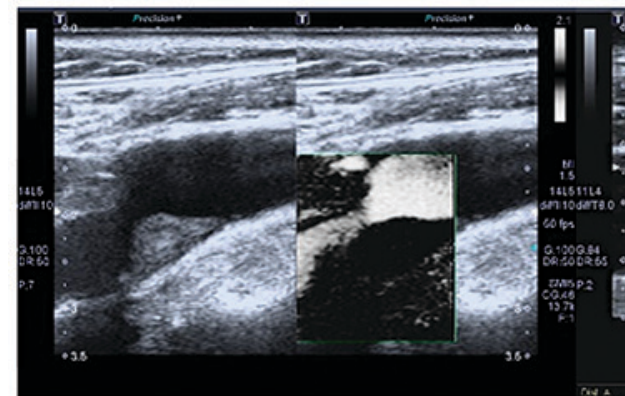

SMI: Grade 0

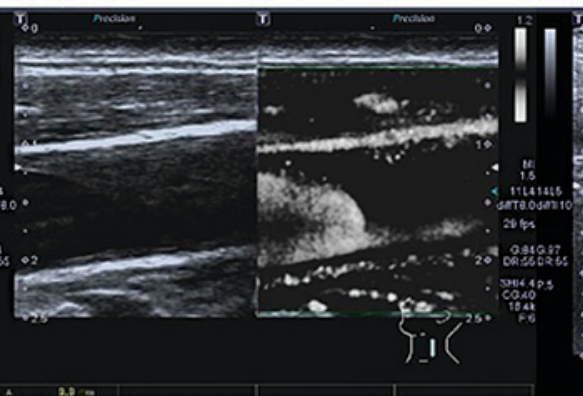

SMI: Grade I

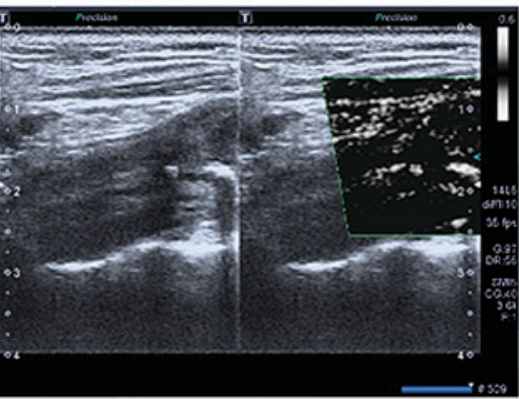

SMI: Grade II

Figure 1. Grading results of SMI. SMI, ultrasound micro-flow imaging.

Table I. Grading results of CEUS.

\begin{tabular}{lcccc}
\hline & \multicolumn{4}{c}{ SMI grading } \\
\cline { 2 - 5 } CEUS grading & 0 & I & II & Total \\
\hline 0 & 7 & 0 & 0 & 7 \\
I & 1 & 6 & 2 & 9 \\
II & 0 & 0 & 23 & 23 \\
Total & 8 & 6 & 25 & 39 \\
\hline
\end{tabular}

$\kappa$ test result: $\kappa=0.860>0$. CEUS, contrast-enhanced ultrasound; SMI, ultrasound micro-flow imaging.

Table II. Neovascularization density of CD34 immunohistochemical staining.

\begin{tabular}{lc}
\hline SMI grading & Neovascularization density $\left(\mathrm{mm}^{2}\right)$ \\
\hline Grade 0 & $1.79 \pm 0.41$ \\
Grade I & $2.26 \pm 0.73$ \\
Grade II & $3.11 \pm 0.48$
\end{tabular}

One-way ANOVA $\mathrm{P}<0.001$; there was no significant difference between Grade 0 and Grade I SMI (P0-I=0.836), while there were significant differences between the remaining 2 groups ( $\mathrm{P} 0-\mathrm{II}=0.001$, PI-II=0.041). SMI, ultrasound micro-flow imaging.

and $92.3 \%$ (12/13), respectively. However, one homogeneous hypoechoic plaque showed no enhancement, and the enhancement scores of the homogeneous hyperechoic plaque group and heterogeneous hyperechoic plaque group were 1 point, accounting for approximately $58.8 \%(10 / 17)$ and $46.2 \%(6 / 13)$, respectively.

CEUS parameters of the 4 groups of plaques. Grading results of 64 plaques of the 39 patients are shown in Table IV. There were 5 plaques with no enhancement in CEUS. Therefore, 59 plaques were included in the CEUS quantitative analysis for the intensities and densities of the 4 groups of plaques according to different echo types, the results showed that the enhancement intensity was increased in the order of homogeneous hyperechoic plaques, heterogeneous hyperechoic
Table III. Grading of CEUS visual scores of carotid atherosclerotic plaques.

\begin{tabular}{lcc}
\hline CEUS grading & No. of plaques & Percentage \\
\hline 0 & 5 & 7.9 \\
1 & 23 & 35.9 \\
2 & 23 & 35.9 \\
3 & 13 & 20.3 \\
Total & 64 & 100 \\
\hline
\end{tabular}

CEUS, contrast-enhanced ultrasound.

plaques, homogeneous hypoechoic plaques, and heterogeneous hypoechoic plaques. The enhancement density was increased in the order of heterogeneous hyperechoic plaques, homogeneous hyperechoic plaques, homogeneous hypoechoic plaques, and heterogeneous hypoechoic plaques. The $\mathrm{H}$ test showed that there were statistically significant differences in the enhancement intensity among the 4 groups $\left(\chi^{2}=29.025, \mathrm{P}<0.001\right)$. Pairwise comparisons showed that there were statistically significant differences between the homogeneous hypoechoic plaque group and homogeneous hyperechoic plaque group and heterogeneous hyperechoic plaque group $(\mathrm{P}<0.05)$. There were statistically significant differences between the heterogeneous hypoechoic plaque group and homogeneous hyperechoic plaque group and heterogeneous hyperechoic plaque group $(\mathrm{P}<0.05)$. There were no statistically significant differences between the other groups (Tables IV and V).

Grading results of SMI and CEUS. The $\kappa$ test result was $\kappa=0.860>0$, indicating that SMI and CEUS have good consistency for the detection of neovascularization in plaques (Table V).

Quantitative parameters of CEUS visual score enhancement. There were 5 plaques with no enhancement in CEUS with 0 points. Therefore, 59 plaques with $>0$ points were included in the CEUS quantitative analysis. The enhanced intensity of CEUS visual scores is shown in Table VI. The visual score of intensity was increased from 1 point, 2 points, and 3 points in turn. There were statistically significant differences in the enhanced intensity values with different scores $\left(\chi^{2}=23.709\right.$, $\mathrm{P}<0.001)$. Pairwise comparison showed that there were 
Table IV. Comparisons of enhanced intensity values of carotid plaques with different echo types: median (interquartile range).

Echo type Median (interquartile range) (x10-5AU)

Homogeneous hypoechoic plaque (Group 1)

$0.0510(0.0269-0.1877)$

Heterogeneous hypoechoic plaque (Group 2)

$0.1021(0.0679-0.2013)$

Homogeneous hyperechoic plaque (Group 3)

$0.0125(0.0036-0.0352)^{\mathrm{a}, \mathrm{b}}$

Heterogeneous hyperechoic plaque (Group 4)

$0.0137(0.0032-0.0300)^{\mathrm{a}, \mathrm{b}}$

$\chi^{2}=29.025, \mathrm{P}<0.001$; the difference was statistically significant compared with ${ }^{\mathrm{a} G r o u p ~} 1$ and ${ }^{\mathrm{b}}$ Group 2 .

Table V. CEUS visual scores of carotid atherosclerotic plaques with different echo types (n).

\begin{tabular}{lccccc}
\hline Echo type & No. of plaques & 0 & 1 & 2 & 3 \\
\hline Homogeneous hypoechoic plaque & 21 & 1 & 6 & 7 & 7 \\
Heterogeneous hypoechoic plaque & 13 & 0 & 1 & 7 & 5 \\
Homogeneous hyperechoic plaque & 17 & 2 & 10 & 5 & 0 \\
Heterogeneous hyperechoic plaque & 13 & 2 & 6 & 4 & 1 \\
\hline
\end{tabular}

CEUS, contrast-enhanced ultrasound.

Table VI. Comparisons of enhanced intensity of carotid atherosclerotic plaques with different scores [median (interquartile range)].

\begin{tabular}{lcc}
\hline Score & No. of plaques & $\begin{array}{c}\text { Median (interquartile range) } \\
(\mathrm{x} 10-5 \mathrm{AU})\end{array}$ \\
\hline 1 & 23 & $0.0086(0.0032-0.0324)$ \\
2 & 23 & $0.0546(0.0300-0.1019)$ \\
3 & 13 & $0.1021(0.0478-0.3468)$
\end{tabular}

statistically significant differences in the enhanced intensity values between the visual score of 1 point and the visual score of 2 and 3 points $(\mathrm{P}<0.05)$, and there was no statistically significant difference between the other groups.

Comparison of the consistency of SMI and CEUS for the diagnosis of neovascularization in plaques. The sensitivity, specificity, and accuracy of SMI for the detection of neovascularization in carotid plaques were 100, 65.0 and $68.8 \%$, respectively. The comparison of the consistency of SMI and CEUS for the diagnosis of neovascularization in plaques showed $\kappa=0.754$ and $\mathrm{P}<0.05$ (Table VII).

\section{Discussion}

Carotid atherosclerotic disease is one of the main causes of cerebral stroke. Histological and morphological studies suggest that carotid plaque-induced stroke depends on whether severe arterial stenosis leads to intracranial hypoperfusion or plaque rupture and ulceration, causing arterial thrombosis, which is referred to as the stability of plaques (12). Therefore, the search for accurate diagnostic and preventive methods, and a risk stratification and treatment plan to reduce the incidence and severity of acute cerebrovascular diseases, should no longer be limited to assessing the degree of arterial stenosis (13). In recent years, it has been shown that hypoechoic plaques share the pathological features of unstable plaques, including large lipid nuclei, thin fibrous caps, a large number of inflammatory cells, and the presence of neovascularization. Hemorrhage and rupture of these plaques occur more easily, resulting in a series of clinical symptoms. Hyperechoic plaques are less prone to rupture because of increased fiber composition and thicker fibrous caps (14-18). According to De Blois et al (19), intima-media thickness and plaque composition are evaluation indicators that can predict the occurrence of cardiovascular and cerebrovascular diseases. Therefore, the accurate evaluation of plaque stability is of great significance for predicting the risk of plaque rupture, and reducing the incidence of acute cerebrovascular disease.

Atherosclerotic plaques are primarily composed of a fibrous cap, lipid necrotic core, inflammatory cells, adventitia, and neovascularization (20-24). Sluimer et al (25) found that hypoxia in plaques is related to thrombosis, atherosclerosis, vascular endothelial growth factor, CD68 expression, and hypoxia-inducible factor. The mRNA and protein expression of hypoxia-inducible factor, target genes, and microvessel density are gradually increased from the early to the stable stage of the lesion, indicating the correlation between the development of hypoxia and atherosclerosis and neovascularization in plaques. New capillaries in plaques, and proliferation of adventitial vasa vasorum or 'vessels of the vessels' are important features of vulnerable plaques, and are closely related to plaque stability. Increased neovascularization is associated with poorer plaque stability, and can more easily result in rupture, causing embolism (26). Therefore, it is highly important to detect and screen for dangerous lesions, 
Table VII. Comparison of the consistency of SMI and CEUS for the detection of neovascularization in plaques.

Detection of neovascularization

in plaques via SMI

Item

Detected

Not detected

Total

Detection of neovascularization in plaques via CEUS

Detected

Not detected

44

7

13

$\kappa$-value

0

P-value

0.754

$<0.05$

SMI, ultrasound micro-flow imaging; CEUS, contrast-enhanced ultrasound.

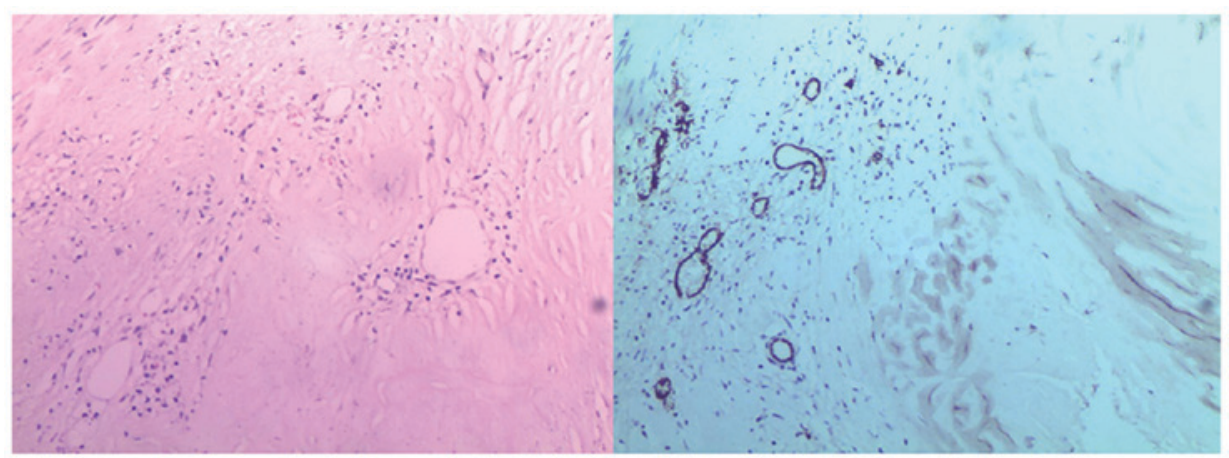

\section{Conventional H\&E staining CD34 immunohistochemical staining}

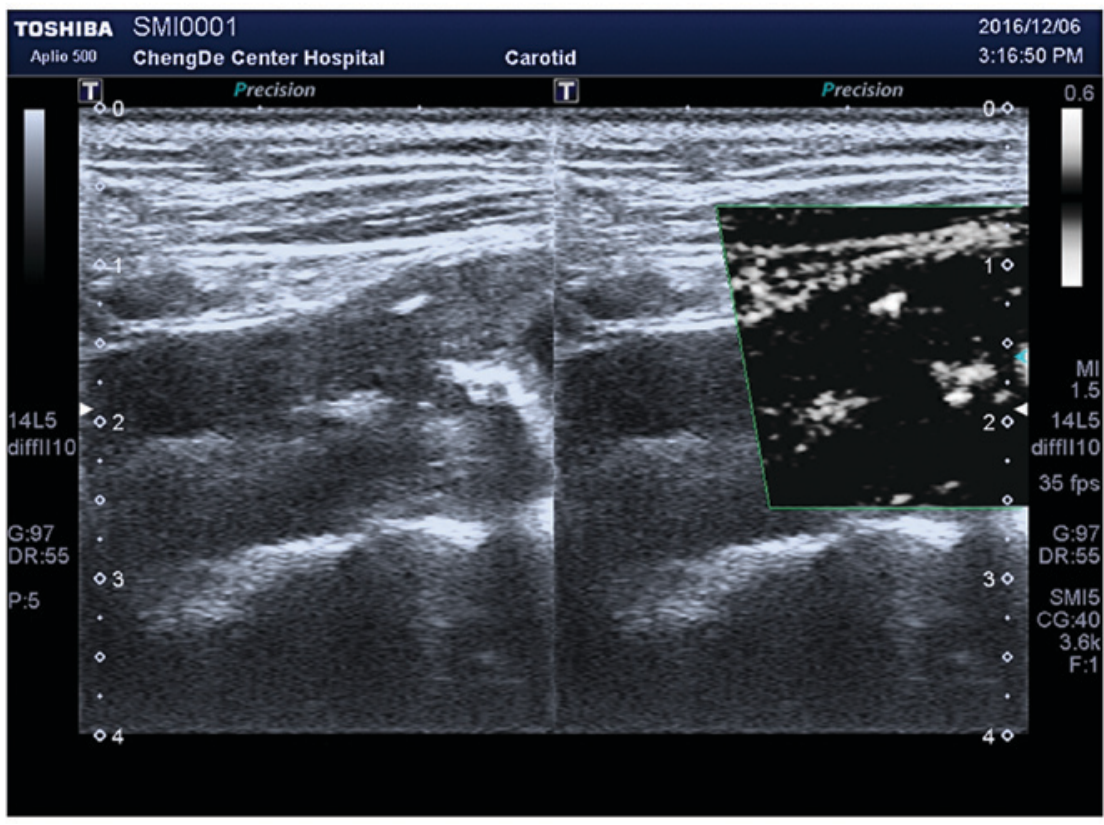

Figure 2. Display of calcification in plaques under SMI mode. SMI, ultrasound micro-flow imaging; H\&E, hematoxylin and eosin.

perform qualitative and quantitative analysis of the proliferative degree of neovascularization in plaques with different echo types, conduct risk stratification for plaques, predict plaque rupture risk, and provide a valuable clinical basis for the prevention and treatment of atherosclerosis-related cardiovascular and cerebrovascular diseases. At present, various imaging methods, such as three-dimensional computer tomography, high-resolution magnetic resonance imaging, and CEUS, have been used to assess the properties of plaques, and the application of SMI in evaluating neovascularization in atherosclerotic plaques has great clinical value (27). Our study showed that the sensitivity, specificity, and accuracy of SMI for the detection of neovascularization in carotid plaques were $100,65.0$, and $68.8 \%$, respectively. The comparison of consistency of SMI and CEUS for the diagnosis of neovascularization in plaques showed $\kappa=0.754$ and $\mathrm{P}<0.05$, suggesting 


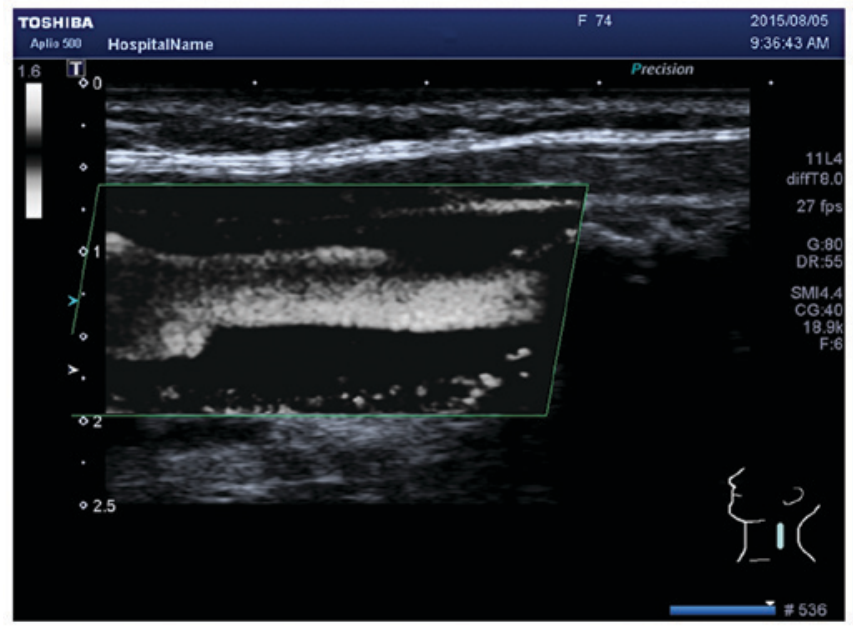

Figure 3. Clear display of neovascularization under SMI mode. SMI, ultrasound micro-flow imaging.

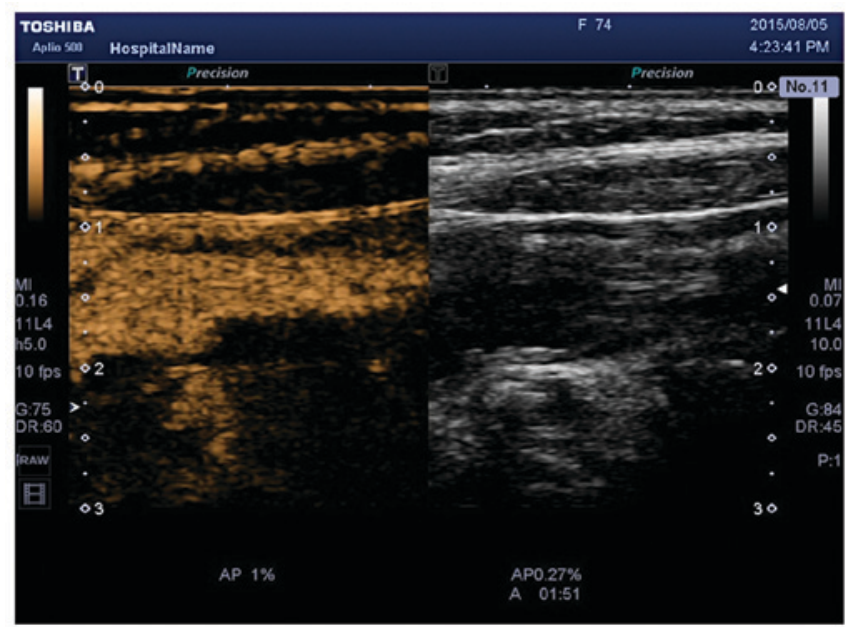

Figure 4. Unclear display of neovascularization within the same plaque shown in Fig. 2 under CEUS mode. CEUS, contrast-enhanced ultrasound.

that SMI has a better power for the diagnosis of neovascularization in plaques, and has good consistency compared with CEUS.

To the best of our knowledge, there are no relevant studies on the detection of neovascularization in carotid plaques via SMI, although there are many studies on other diseases that indicate that SMI can effectively detect blood vessels $(27,28)$. In this study, we found that 1 plaque had Grade 0 SMI and Grade I CEUS, and 2 plaques had Grade II SMI and Grade I CEUS. The optimal debugging condition of SMI therefore requires further study, as it is a new technique for observing neovascularization. When using the SMI mode to observe neovascularization in plaques, calcification in plaques shows a similar performance to neovascularization in the SMI mode. However, it can distinguish between them. New vessels will flow with time, but the development of calcification is static, brighter than new blood vessels, and can coincide with twodimensional images (Fig. 2). Therefore, it can eliminate the impact of calcification on the observation of neovascularization. Compared to CEUS, the SMI technique uses a new self-adaptive algorithm to identify and eliminate tissue motion

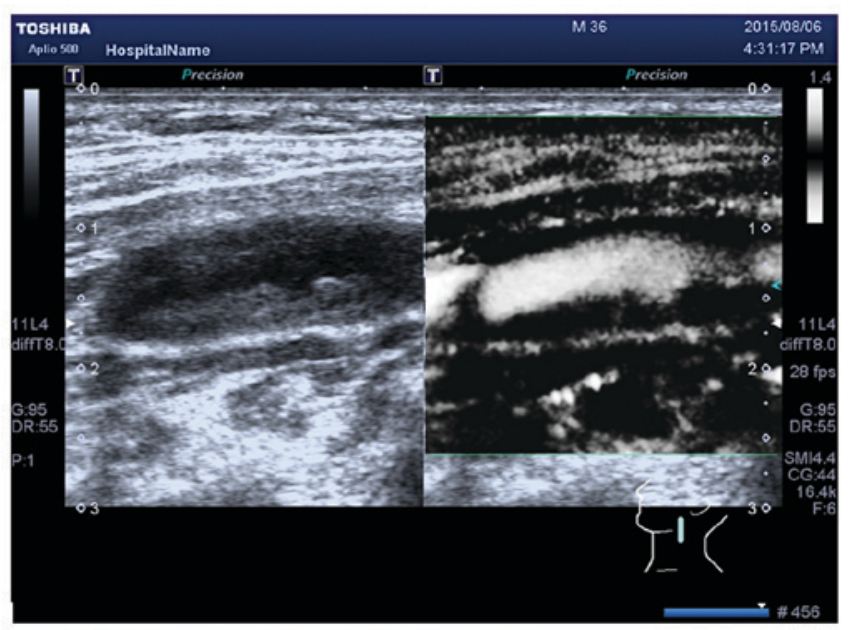

Figure 5. Grade I neovascularization within the ulcer plaque under SMI mode. SMI, ultrasound micro-flow imaging.

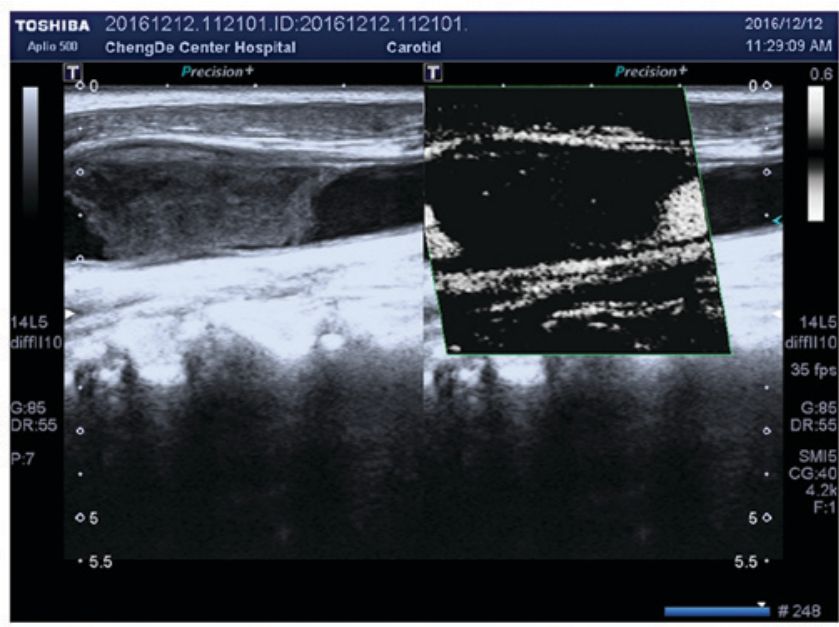

Figure 6. Grade II neovascularization within the hypoechoic plaque under SMI mode. SMI, ultrasound micro-flow imaging.

artifacts, retain the most subtle low-velocity blood flow signals, and can show unparalleled details with high definition and clear neovascularization in plaques (Figs. 3 and 4).

Before SMI, CEUS was commonly used to observe neovascularization in carotid plaques, but the technique is invasive and limited by the physical condition of the patient, with relatively high costs. During routine ultrasound, we used the SMI technique to evaluate neovascularization in plaque development. The SMI procedure is basically the same as color Doppler flow imaging without the waiting time for contrast agent to distribute to vessels. Therefore, the time necessary for observing neovascularization is much shorter than that of CEUS, reducing the psychological and financial burden of patients, and reducing the workload of ultrasound physicians. Patients with ulcerated plaques received SMI to observe neovascularization in carotid plaques, followed by risk stratification for ulcerated plaques, and prediction of the risk of stroke. As shown in Figs. 5 and 6, the plaques in patients with ultra-low echo plaques can be deformed with the heart, which may be because of plaque rupture, causing thrombosis. SMI examination identified the visible blood 
flow signals in ultra-low echo, which may have occurred because most of the components of the plaques were liquid. Regarding the detection of carotid plaques, when the common carotid artery bifurcation site or plaque site is relatively high, the vascular probe cannot meet the needs of clinical examination. Therefore, a conventional abdominal probe should be used. The SMI technique is not limited by the probe. Given that the SMI technique is non-invasive with easy operation and no additional injury in routine ultrasound examination, it is suitable for the evaluation of carotid atherosclerotic plaques.

The results of our study showed that the GSM values were increased in the order of homogeneous hypoechoic plaques (34.78 \pm 11.16$)$, heterogeneous hypoechoic plaques $(44.55 \pm 11.97)$, homogeneous hyperechoic plaques $(50.20 \pm 13.61)$, and heterogeneous hyperechoic plaques $(77.50 \pm 16.04)$. Moreover, the differences in GSM values between the 4 groups of plaques were statistically significant $(\mathrm{F}=29.365, \mathrm{P}<0.001)$, indicating that lower plaque echo is associated with lower GSM value, which is consistent with the previous histological study and GSM correspondence analysis (29). The GSM values of the heterogeneous hypoechoic plaque group were higher than those of the homogeneous hypoechoic plaque group. The GSM values of the heterogeneous hyperechoic plaque group were higher than those of the homogeneous hyperechoic plaque group, and the differences in the comparisons between the two groups were statistically significant $(\mathrm{P}<0.05)$. These observations were related to the histological composition and classification of plaques: hypoecho prevailed in heterogeneous hypoechoic plaques, and the calcium and fiber composition in the hyperechoic area $(<25 \%$ of the total plaque area) made the GSM values higher than those of the homogeneous hypoechoic plaque group; hyperecho prevailed in heterogeneous hyperechoic plaques, and the hypoechoic and echoless areas $(<25 \%$ of the total plaque area) could decrease the GSM value of plaques; however, the hyperechoic area in heterogeneous hyperechoic plaques accounted for the majority, and two-dimensional ultrasonography showed that its gray-scale intensity was higher than that of the homogeneous hypoechoic plaque group. According to ultrasound imaging and the acoustic impedance principle, combined with pathological tissue components, it may be related to a higher calcareous component and higher GSM value. Therefore, the GSM values of the heterogeneous hyperechoic plaque group were higher than those of the homogeneous hyperechoic plaque group. In addition, the differences between the heterogeneous hypoechoic and homogeneous hyperechoic plaque groups were not statistically significant $(P=0.245)$, possibly because the calcareous component in heterogeneous hypoechoic plaques increases the GSM value, thus it is close to the GSM value of homogeneous hyperechoic plaques containing fibrous tissue components. The GSM technique provides a quantitative and objective evaluation method for the classification of plaques with different echo types, avoiding the subjectivity of judging the plaque echo types by naked eye evaluation. Therefore, the repeatability is high (30-33). According to Mathiesen et al (34), hypoechoic plaques can increase the risk of ischemic cerebrovascular events, which is significantly correlated with the plaque echo type $(\mathrm{P}=0.015)$, and detecting the plaque echo type via two-dimensional ultrasound can predict the risk of ischemic cerebrovascular events.
In this study, $\kappa$ test results showed that SMI and CEUS have good consistency for the detection of neovascularization in plaques. One-way ANOVA showed that SMI can effectively distinguish neovascularization density. However, there was no statistically significant difference between Grade 0 and Grade 1 SMI. The rank-sum test and Spearman's rank correlation test showed that SMI grading can predict the occurrence of stroke to some extent. Higher SMI grading is associated with higher risk of stroke.

In conclusion, our results suggest that the GSM technique can evaluate plaque stability through the quantitative classification of plaque echoes, for use as an important reference index for predicting the risk of stroke. This is consistent with the study by Ariyoshi et al (35), who measured the plaque echoes of carotid atherosclerotic plaques in 84 patients with type 2 diabetes mellitus using GSM, and investigated the correlations between different echoes and different GSM values, and the incidence rate of cardiovascular and cerebrovascular events. The GSM technique can be used in combination with CEUS to directly observe the proliferation of neovascularization in plaques, and obtain significant quantitative parameters, to evaluate the stability of plaque more objectively.

\section{References}

1. Gray-Weale AC, Graham JC, Burnett JR, Byrne K and Lusby RJ: Carotid artery atheroma: comparison of preoperative B-mode ultrasound appearance with carotid endarterectomy specimen pathology. J Cardiovasc Surg (Torino) 29: 676-681, 1988.

2. Naghavi M, Libby P, Falk E, Casscells SW, Litovsky S, Rumberger J, Badimon JJ, Stefanadis C, Moreno P, Pasterkamp G, et al: From vulnerable plaque to vulnerable patient: a call for new definitions and risk assessment strategies: part I. Circulation 108: 1664-1672, 2003.

3. Hellings WE, Peeters W, Moll FL, Piers SR, van Setten J, Van der Spek PJ, de Vries JP, Seldenrijk KA, De Bruin PC, Vink A, et al: Composition of carotid atherosclerotic plaque is associated with cardiovascular outcome: a prognostic study. Circulation 121: 1941-1950, 2010.

4. Hingwala D, Kesavadas C, Sylaja PN, Thomas B and Kapilamoorthy TR: Multimodality imaging of carotid atherosclerotic plaque: going beyond stenosis. Indian J Radiol Imaging 23: 26-34, 2013.

5. Fleiner M, Kummer M, Mirlacher M, Sauter G, Cathomas G, Krapf R and Biedermann BC: Arterial neovascularization and inflammation in vulnerable patients: early and late signs of symptomatic atherosclerosis. Circulation 110: 2843-2850, 2004.

6. Shah F, Balan P, Weinberg M, Reddy V, Neems R, Feinstein M, Dainauskas J, Meyer P, Goldin M and Feinstein SB: Contrastenhanced ultrasound imaging of atherosclerotic carotid plaque neovascularization: a new surrogate marker of atherosclerosis? Vasc Med 12: 291-297, 2007.

7. Virmani R, Kolodgie FD, Burke AP, Finn AV, Gold HK, Tulenko TN, Wrenn SP and Narula J: Atherosclerotic plaque progression and vulnerability to rupture: angiogenesis as a source of intraplaque hemorrhage. Arterioscler Thromb Vasc Biol 25: 2054-2061, 2005.

8. Staub D, Partovi S, Schinkel AF, Coll B, Uthoff H, Aschwanden M, Jaeger KA and Feinstein SB: Correlation of carotid artery atherosclerotic lesion echogenicity and severity at standard US with intraplaque neovascularization detected at contrast-enhanced US. Radiology 258: 618-626, 2011.

9. Russell DA, Wijeyaratne SM and Gough MJ: Changes in carotid plaque echomorphology with time since a neurologic event. J Vasc Surg 45: 367-372, 2007.

10. Sztajzel R, Momjian S, Momjian-Mayor I, Murith N, Djebaili K, Boissard G, Comelli M and Pizolatto G: Stratified gray-scale median analysis and color mapping of the carotid plaque: correlation with endarterectomy specimen histology of 28 patients. Stroke 36: 741-745, 2005. 
11. Nakamura J, Nakamura T, Deyama J, Fujioka D, Kawabata K, Obata JE, Watanabe K, Watanabe $\mathrm{Y}$ and Kugiyama K: Assessment of carotid plaque neovascularization using quantitative analysis of contrast-enhanced ultrasound imaging is useful for risk stratification in patients with coronary artery disease. Int J Cardiol 195: 113-119, 2015.

12. Fisher M, Paganini-Hill A, Martin A, Cosgrove M, Toole JF, Barnett HJ and Norris J: Carotid plaque pathology: thrombosis, ulceration, and stroke pathogenesis. Stroke 36: 253-257, 2005.

13. ten Kate GL, van Dijk AC, van den Oord SC, Hussain B, Verhagen HJ, Sijbrands EJ, van der Steen AF, van der Lugt A and Schinkel AF: Usefulness of contrast-enhanced ultrasound for detection of carotid plaque ulceration in patients with symptomatic carotid atherosclerosis. Am J Cardiol 112: 292-298, 2013.

14. Vicenzini E, Giannoni MF, Sirimarco G, Ricciardi MC, Toscano M, Lenzi GL and Di Piero V: Imaging of plaque perfusion using contrast-enhanced ultrasound - clinical significance. Perspect Med 1: 44-50, 2012.

15. Hoogi A, Adam D, Hoffman A, Kerner H, Reisner S and Gaitini D: Carotid plaque vulnerability: quantification of neovascularization on contrast-enhanced ultrasound with histopathologic correlation. AJR Am J Roentgenol 196: 431-436, 2011

16. Nandalur KR, Baskurt E, Hagspiel KD, Phillips CD and Kramer CM: Calcified carotid atherosclerotic plaque is associated less with ischemic symptoms than is noncalcified plaque on MDCT. AJR Am J Roentgenol 184: 295-298, 2005.

17. Seo Y, Watanabe S, Ishizu T, Moriyama N, Takeyasu N, Maeda H, Ishimitsu T, Aonuma K and Yamaguchi I: Echolucent carotid plaques as a feature in patients with acute coronary syndrome. Circ J 70: 1629-1634, 2006.

18. Grønholdt ML, Nordestgaard BG, Schroeder TV, Vorstrup S and Sillesen H: Ultrasonic echolucent carotid plaques predict future strokes. Circulation 104: 68-73, 2001.

19. De Blois J, Stranden E, Jogestrand T, Henareh L and Agewall S: Echogenicity of the carotid intima-media complex and cardiovascular risk factors. Clin Physiol Funct Imaging 32: 400-403, 2012.

20. Shalhoub J, Owen DR, Gauthier T, Monaco C, Leen EL and Davies AH: The use of contrast enhanced ultrasound in carotid arterial disease. Eur J Vasc Endovasc Surg 39: 381-387, 2010

21. Ogata T, Yasaka M, Wakugawa Y, Kitazono T and Okada Y: Morphological classification of mobile plaques and their association with early recurrence of stroke. Cerebrovasc Dis 30: 606-611, 2010

22. Higashida T, Kanno H, Nakano M, Funakoshi K and Yamamoto I Expression of hypoxia-inducible angiogenic proteins (hypoxiainducible factor-1alpha, vascular endothelial growth factor, and E26 transformation-specific-1) and plaque hemorrhage in human carotid atherosclerosis. J Neurosurg 109: 83-91, 2008.

23. Lee SH, Lee JH, Yoo SY, Hur J, Kim HS and Kwon SM: Hypoxia inhibits cellular senescence to restore the therapeutic potential of old human endothelial progenitor cells via the hypoxia-inducible factor-1 $\alpha$-TWIST-p21 axis. Arterioscler Thromb Vasc Biol 33: 2407-2414, 2013
24. Kim HS, Woo JS, Kim BY, Jang HH, Hwang SJ, Kwon SJ, Choi EY, Kim JB, Cheng X, Jin E, et al: Biochemical and clinical correlation of intraplaque neovascularization using contrast-enhanced ultrasound of the carotid artery. Atherosclerosis 233: 579-583, 2014.

25. Sluimer JC, Gasc JM, van Wanroij JL, Kisters N, Groeneweg M, Sollewijn Gelpke MD, Cleutjens JP, van den Akker LH, Corvol P, Wouters BG, et al: Hypoxia, hypoxia-inducible transcription factor, and macrophages in human atherosclerotic plaques are correlated with intraplaque angiogenesis. J Am Coll Cardiol 51: 1258-1265, 2008.

26. Zhao X, Kong J, Zhao Y, Wang X, Bu P, Zhang C and Zhang Y: Gene silencing of TACE enhances plaque stability and improves vascular remodeling in a rabbit model of atherosclerosis. Sci Rep 5: 17939, 2015.

27. Wang YH, Zheng QC, Yuan GX and Ren JH: The application of ultrasound B-flow imaging in detection of carotid atherosclerotic micro-vessel with ischemic cerebrovascular disease. Saudi Med J 33: 1080-1086, 2012

28. Lind L, Andersson J, Rönn M, Gustavsson T, Holdfelt P, Hulthe J, Elmgren A, Zilmer K and Zilmer M: Brachial artery intima-media thickness and echogenicity in relation to lipids and markers of oxidative stress in elderly subjects: the prospective investigation of the vasculature in Uppsala Seniors (PIVUS) Study. Lipids 43: 133-141, 2008.

29. Schulte-Altedorneburg G, Droste DW, Haas N, Kemény V, Nabavi DG, Füzesi L and Ringelstein EB: Preoperative B-mode ultrasound plaque appearance compared with carotid endarterectomy specimen histology. Acta Neurol Scand 101: 188-194, 2000 .

30. Ostling G, Hedblad B, Berglund G and Gonçalves I: Increased echolucency of carotid plaques in patients with type 2 diabetes. Stroke 38: 2074-2078, 2007.

31. Sabetai MM, Tegos TJ, Nicolaides AN, Dhanjil S, Pare GJ and Stevens JM: Reproducibility of computer-quantified carotid plaque echogenicity: can we overcome the subjectivity? Stroke 31: 2189-2196, 2000.

32. Kyriacou EC, Petroudi S, Pattichis CS, Pattichis MS, Griffin M, Kakkos S and Nicolaides A: Prediction of high-risk asymptomatic carotid plaques based on ultrasonic image features. IEEE Trans Inf Technol Biomed 16: 966-973, 2012.

33. Östling G, Persson M, Hedblad B and Gonçalves I: Comparison of grey scale median (GSM) measurement in ultrasound images of human carotid plaques using two different softwares. Clin Physiol Funct Imaging 33: 431-435, 2013.

34. Mathiesen EB, Bønaa KH and Joakimsen O: Echolucent plaques are associated with high risk of ischemic cerebrovascular events in carotid stenosis: the troms $\varnothing$ study. Circulation 103: 2171-2175, 2001.

35. Ariyoshi K, Okuya S, Kunitsugu I, Matsunaga K, Nagao Y, Nomiyama R, Takeda K and Tanizawa Y: Ultrasound analysis of gray-scale median value of carotid plaques is a useful reference index for cerebro-cardiovascular events in patients with type 2 diabetes. J Diabetes Investig 6: 91-97, 2015. 